\title{
A NEW TYPE OF ASYMMETRIC OUTFLOW IN THE RECURRENT NOVA RS OPH IN QUIESCENCE
}

\author{
T. IIJIMA \\ Astronomical Observatory of Padova, Asiago Section \\ Osservatorio Astrofisico, I-36012 Asiago (Vi), Italy
}

\begin{abstract}
H I emission lines in the spectrum of the recurrent nova and symbiotic star RS Oph in quiescence are sometimes accompanied by blueor red-shifted broad emission components. The displacements of the broad components from the narrow lines change from -1300 to $+1200 \mathrm{~km} \mathrm{~s}^{-1}$, but possibly not continuously. The broad components seem to appear in some restricted regions on the spectra. An asymmetry between the blue- and red-shifts is noticed.
\end{abstract}

RS Oph is a binary system consisting of a white dwarf and a masslosing M type giant (e.g. Bruch 1986; Garcia 1986; Dobrzycka \& Kenyon 1994). Recently, broad emission components of $\mathrm{H}$ I lines were found in some quiescent spectra, and their peculiar properties have been determined, e.g. they appear on the blue side of the narrow lines or on the red side, but not on both sides simultaneously; furthermore, their wavelengths, profiles, and intensities change with a time-scale of days (Iijima et al. 1994; hereafter Paper I). Here results of new observations in 1993 are reported. The criteria of the observations are the same as in Paper I. The broad components usually show double-peaked profiles. The last five columns of Table 1 give displacements of individual peaks of the broad components of $\mathrm{H} \alpha$ and $\mathrm{H} \beta$ relative to the narrow lines and of the center wavelengths between the two peaks $v_{\text {cent }}$. As in Paper I, the more distant peak is called the primary peak. The observational error is about $\pm 30 \mathrm{~km} \mathrm{~s}^{-1}$. A histogram of $v_{\text {cent }}$ is shown in Fig. 1, where the results presented in Paper I are also included. Since there is a strong narrow emission line at the center, displacements less than $\pm 800 \mathrm{~km} \mathrm{~s}^{-1}$ are not detectable. As seen in the figure, there are four prominent peaks in the histogram located at, from left to right, $-1230 \pm 25,-1140 \pm 15,+930 \pm 20$, and $+1020 \pm 20 \mathrm{~km} \mathrm{~s}^{-1}$. It seems that the broad components do not move continuously, but appear in these restricted

\section{1}

A. Evans and J. H. Wood (eds.), Cataclysmic Variables and Related Objects, 331-332.

(c) 1996 Kluwer Academic Publishers. Printed in the Netherlands. 
TABLE 1. Spectroscopic observations of RS Oph in 1993

\begin{tabular}{lrccccccc}
\hline Date & $\begin{array}{r}\text { Exp. } \\
(\mathrm{m})\end{array}$ & $\begin{array}{c}\mathrm{JD} \\
-2440000\end{array}$ & $\begin{array}{c}\text { Spectral range } \\
(\mathrm{nm})\end{array}$ & $\mathrm{H} \alpha^{p}$ & $\mathrm{H} \alpha^{s}$ & $\begin{array}{c}\mathrm{H} \beta^{p} \\
\left(\mathrm{~km} \mathrm{~s}^{-1}\right)\end{array}$ & $\mathrm{H} \beta^{s}$ & $v_{\text {cent }}$ \\
\hline July 28 & 20 & 9197.45 & $398-510$ & & & -1405 & -733 & -1069 \\
July 28 & 5 & 9197.48 & $571-683$ & -1496 & -1014 & & & -1255 \\
Aug. 13 & 20 & 9213.41 & $392-504$ & & & -1540 & -769 & -1155 \\
Aug. 13 & 5 & 9213.45 & $580-692$ & -1514 & -993 & & & -1254 \\
Aug. 14 & 20 & 9214.41 & $396-508$ & & & -1458 & -843 & -1150 \\
Aug. 14 & 5 & 9214.45 & $574-686$ & -1508 & -976 & & & -1242 \\
\hline
\end{tabular}

Exp.: Exposure time; $p$ : primary peak, $s$ : secondary peak, $v_{\text {cent }}$ : mean of $p$ and $s$

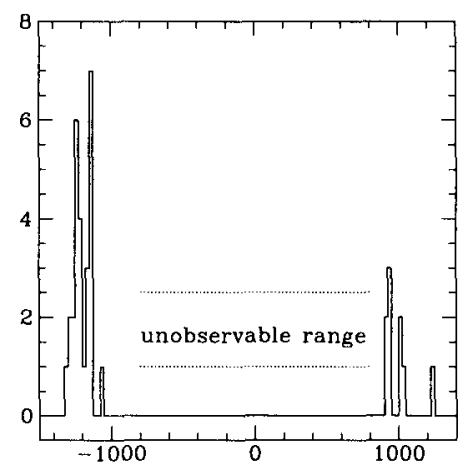

Figure 1. Histogram of radial velocities of the center of broad emission components. Horizontal scale in $\mathrm{km} \mathrm{s}^{-1}$.

regions. The lack of blue-shifts around $-1000 \mathrm{~km} \mathrm{~s}^{-1}$ is noticeable. This result suggests that there is an asymmetry between the blue- and redshifts, or the center of the symmetry is blue-shifted by about $100 \mathrm{~km} \mathrm{~s}^{-1}$. It seems difficult to explain these asymmetric and discrete displacements with known models of bipolar gas outflow. There might be a new type of gas outflow in this system. Among numerous symbiotic stars only this object and CH Cyg (Paper I; Kuczawska, Mikolajewski \& Kirejczyk 1992; Leedjärv 1993) have similar broad emissions.

\section{References}

Bruch A., 1986, A\&A, 167, 91

Dobrzycka D., Kenyon S.J., 1994, AJ, 108, 2259

Garcia M.R., 1986, AJ, 91, 1400

Iijima T., Strafella F., Sabbadin F., Bianchini A., 1994, A\&A, 283, 919

Kuczawska E., Mikolajewski M., Kirejczyk K., 1992, IBVS 3806

Leedjärv L., 1993, IBVS 3951 